\title{
IN VITRO REACTIVATION OF ACETYLCHOLINESTERASE INHIBITED BY CYCLOSARIN USING BISQUATERNARY PYRIDINIUM ALDOXIMES K005, K033, K027 AND K048
}

\author{
Kamil Kuča, Lucie Ševelová-Bartošová, Gabriela Krejčová-Kunešová
}

\author{
Purkyně Military Medical Academy, Hradec Králové, Czech Republic: Department of Toxicology
}

Summary: We have tested four new bisquaternary pyridinium acetylcholinesterase (AChE; EC 3.1.1.7) reactivators - K005 (1,3-bis(2-hydroxyiminomethylpyridinium) propane dibromide), K033 (1,4-bis(2-hydroxyiminomethylpyridinium) butane dibromide), K027 (1-(4-hydroxyiminomethylpyridinium)-3-(4-carbamoylpyridinium) propane dibromide) and K048 (1-(4-hydroxyiminomethylpyridinium)-4-(4-carbamoylpyridinium) butane dibromide) as the potential reactivators of AChE inhibited by cyclosarin. Their reactivation potencies were studied using standard in vitro reactivation test. Rat brain homogenate was used as the source of the enzyme. Oxime K033 seems to be the most potent reactivator of cyclosarin-inhibited AChE. Its reactivation potency is significantly higher than the efficacy of all other tested AChE reactivators.

Key words: Cyclosarin; In vitro; Reactivation; Inhibition; Acetylcholinesterase; K005; K033; K027; K048; Oximes; Nerve agents; GF agent; Cyclosin

\section{Introduction}

Sarin (GB; O-isopropylmethylfluorophosphonate), tabun (GA; O-ethyldimethylamidocyanophosphate), soman (GD; O-pinacolylmethylfluorophosphonate), cyclosarin (GF; O-cyclohexylmethylfluorophosphonate) and VX (O-ethyl-S(2-diisopropylaminoethyl)-methylthiophosphonate) belong among the best known nerve agents (20).

These compounds cause longterm inactivation of acetylcholinesterase (AChE, EC 3.1.1.7) and butyrylcholinesterase (BChE, EC 3.1.1.8) by phosphorylating a serine residue at the active site of the enzyme $(8,18)$.

The presently used antidotes against nerve agents intoxications, such as pralidoxime or obidoxime (functional antidotes) in combination with atropine (causal antidote), do not appear as suitable antidotes against poisonings with all kinds of nerve agents $(6,7,9,19)$. For example, pralidoxime has no reactivation ability in treatment of cyclosarin and tabun-inhibited AChE $(11,17)$. Obidoxime is not able to reactivate $\mathrm{AChE}$ inhibited by cyclosarin and its ability to reactivate tabun inhibited AChE is poor (1).

In this work, we are interested in the comparison of four new potential AChE reactivators - K005 (1,3-bis(2-hydroxyiminomethylpyridinium) propane dibromide), K033 (1,4-bis(2-hydroxyiminomethylpyridinium) butane dibromide), K027 (1-(4-hydroxyiminomethylpyridinium)-3-(4carbamoylpyridinium) propane dibromide) and K048 (1-(4-hydroxyiminomethylpyridinium)-4-(4-carbamoylpyridinium) butane dibromide) developed during last year at the Department of Toxicology Purkyne Medical Military Academy (Figure 1). Their synthesis $(10,11,13)$ and reactivation potencies $(12,14,15,16)$ were described earlier in some chemical, biochemical and toxicological journals.

This work summarizes reactivation potency of these drugs as treatment of cyclosarin intoxications in vitro.<smiles></smiles>

Fig. 1: Structures of the tested oximes.

\section{Material and methods}

All new oximes were synthesized previously at our department $(10,11,13)$. Cyclosarin [GF; O-cyclohexylmethylfluorophosphate] of $95 \%$ purity was obtained from the Military Technical Institute (Zemianské Kostolany, Slovak Republic). All other chemicals of a reagent grade were obtained from commercial sources. 
Standard in vitro method was used for the evaluation of reactivation potencies of these new drugs $(14,15)$. General conditions were taken as follows. Rat brain homogenate diluted in distilled water $(10 \%, \mathrm{w} / \mathrm{v})$ was used as a source of AChE. Measurement was taken at $25^{\circ} \mathrm{C}, \mathrm{pH} 8$, and concentration of the AChE reactivators in the scale from $10^{-8}$ to $10^{-2} \mathrm{M}$. The activity of AChE was determined by $\mathrm{pH}$ static titration of acetic acid released from acetylcholine iodide using the autotitrator (Copenhagen, Danmark).

\section{Results}

\section{Affinity to the acetylcholinesterase}

Values of the constant $\mathrm{K}_{\mathrm{DIS}}$ characterizing the affinity of newly synthesized oximes to the intact $\mathrm{AChE}$ are shown in Table 1. As lower its value is as higher the affinity of tested oximes towards the $\mathrm{AChE}$ is. As indicated in the Table 1, oxime K005 has the lowest value of the constant $\mathrm{K}_{\mathrm{DIS}}$. Due to this fact, its affinity towards the enzyme is the highest among all the tested oximes. Value of the affinity of the oxime K033 is comparable with that of oxime K005.

Tab. 1: Affinity of the tested oximes towards acetylcholinesterase.

\begin{tabular}{|c|c|}
\hline Oxime & $K_{D I S}[\mu \mathrm{M}]$ \\
\hline K005 & 53 \\
\hline K033 & 65 \\
\hline K027 & 5888 \\
\hline K048 & 228 \\
\hline
\end{tabular}

\section{Reactivation of the acetylcholinesterase}

Kinetics parameters (dissociation constant: $K_{\mathrm{R}}$ and rate constants: $k_{\mathrm{R}}$ and $k_{\mathrm{r}}$ ) shown in Table 2 characterize the ability of the new oximes to reactivate cyclosarin-inhibited $\mathrm{AChE}$ in vitro. We were not able to measure rate constants of the compounds K027 and K048, due to the very low ability of these oximes reactivate cyclosarin-inhibited AChE.

The values of the constant $K_{R}$ characterizing the affinity of oximes to cyclosarin-inhibited $\mathrm{AChE}$ indicate that the affinity of the compound K005 to the enzyme-inhibitor complex is the highest in the comparison to all new oximes tested.

Tab. 2: Kinetic constants of the reactivation potency of tested oximes.

\begin{tabular}{|l|c|c|c|}
\hline Oxime & $K_{R}[\mu \mathrm{M}]$ & $k_{R}\left[\min ^{-1}\right]$ & $k_{r}\left[\mathrm{M}^{-1} \cdot \min ^{-1}\right]$ \\
\hline K005 & 5 & 0,010 & 2196 \\
\hline K033 & 20 & 0,095 & 4872 \\
\hline K027 & - & - & - \\
\hline K048 & - & - & - \\
\hline
\end{tabular}

$$
(-)=\text { not measurable values }
$$

The values of the constant $k_{\mathrm{r}}$ express the breakdown of the intermediate complex. The highest value of this con- stant was obtained for oxime K033. The values of this constant decrease as follows: K033 > K005 > K027 = K048.

Oxime K033 has the highest bimolecular constant of reactivation $\left(k_{\mathrm{r}}\right)$ representing overall reactivation ability.

The potency of tested oximes to reactivate cyclosarin-inhibited AChE is demonstrated in Figure 2.

In the case of oxime K033, $46 \%$ reactivation of cyclosarin-inhibited $\mathrm{AChE}$ was obtained at the concentration $10^{-4} \mathrm{M}$. Maximal reactivation potency for the oxime K005 (9\%) was obtained at the same concentration of the reactivators. Oxime K048 reached the maximum reactivation potency $11 \%$ at the concentration $10^{-2} \mathrm{M}$. Unfortunately, this concentration is not suitable for human use. Oxime $\mathrm{K} 027$ was not able to reactivate AChE inhibited by cyclosarin. In the case of human relevant concentration $\left[10^{-5}\right]$, the percentage of reactivation of cyclosarin-inhibited AChE does not reach $30 \%$ regardless of the oxime used.

According to our evaluation of all kinetic constants and concentration-reactivation relationship, we can confirm that the best reactivator from the new oximes seems to be oxime K033.

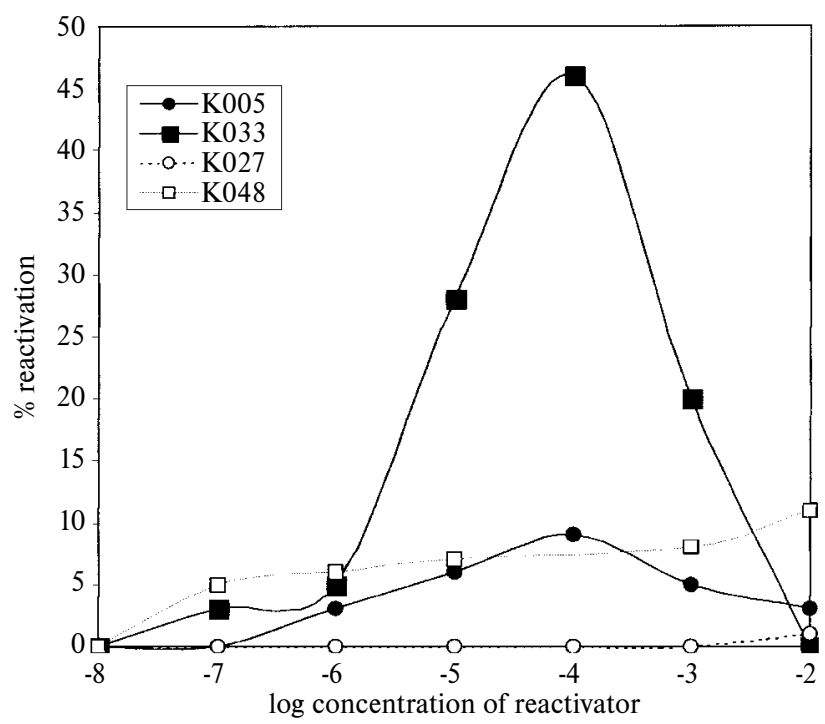

Fig. 2: Concentration versus reactivity relationship of the tested oximes.

\section{Discussion}

Thanks to the fact, that currently used AChE reactivators do not reactivate $\mathrm{AChE}$ inhibited by all kinds of nerve agents, searching for new AChE reactivator, which could reactivate $\mathrm{AChE}$ inhibited by more nerve agents, is needed. In our work, we have presented reactivation potency of new bisquaternary pyridinium AChE reactivators - K005, K033, K027 and K048 in cyclosarin intoxications. Although, this work presents just in vitro experiments our results are important because of the close relationship between in vitro and in vivo results $(4,5)$. 
It is very difficult to find an ideal broad-spectrum reactivator of nerve agents-inhibited AChE. It should have the value of $K_{\mathrm{R}}$ from 10 to 100 times lower than $K_{\mathrm{m}}$ of the native substrate $\left(K_{\mathrm{m}}\right.$ for AChE + acetylcholine iodide is 200 $\mu \mathrm{M})$ and the value of KDIS from 10 to 100 times higher than $K_{\mathrm{m}}(3,4)$. Thus, the affinity of the AChE reactivator to the inhibited enzyme should be higher than the affinity to the reactivated, respectively, intact enzyme. Both oximes K005 and K033 fulfil this rule.

In the case of the oximes $\mathrm{K} 005$ and $\mathrm{K} 033$, the rate constant $\mathrm{k}_{\mathrm{R}}$ is not affected by $\mathrm{pKa}$ of these oximes because of their similar values of this constant (K005 - 7.8; K033 7.7). Their differencies (rate constant $k_{\mathrm{R}}$ ) are caused just thanks to the length of the connecting chain between both pyridinium rings.

Our results presented in this work confirm the fact that the reactivation of inhibited $\mathrm{AChE}$ depends not only on the inhibitors but also on the chemical structure of the reactivators (10). As you can notice in Figure 1, chemical structures of the AChE reactivators $\mathrm{K} 005$ and $\mathrm{K} 033$ differ in the symmetry from the AChE reactivators K027 and K048. Bisquaternary symmetric pyridinium aldoximes K005 and K033 with functional aldoxime groups at both pyridinium rings are better reactivators of AChE inhibited by cyclosarin. On the other hand, asymmetric $\mathrm{AChE}$ reactivators K027 and K048 are not able to reactivate AChE inhibited by this nerve agent. As publicated earlier, these AChE reactivators (K027 and K048) are potent reactivators of AChE inhibited by tabun and sarin $(11,15)$.

Our data confirm earlier mentioned results presented by Dohnal et al. that higher symmetry of the AChE reactivators increases reactivation ability of the oximes (2). These results are in good agreement with work presented by Kassa and Cabal (3). They have evaluated MMC-4 (1,1-bis(4-hydroxyiminomethylpyridinium) methane dibromide), symmetric bisquaternary pyridinium salt with aldoxime groups at the position four, as the best oxime for the reactivation of $\mathrm{AChE}$ inhibited by cyclosarin.

In conclusion, we have tested in vitro four new $\mathrm{AChE}$ reactivators (K005, K033, K027 and K048). Their reactivation potency was studied using cyclosarin-inhibited AChE. K033 seems to be promising reactivator of cyclosarin-inhibited AChE. Its reactivation potency is significantly higher than the efficacy of all other tested AChE reactivators. Thanks to the high reactivation potency of this AChEreactivator, oxime K033 will be soon evaluated in vivo using standard reactivation test (19).

\section{Acknowledgement}

The authors are grateful to Mrs. I. Ježková and Mrs. M. Hrabinová for their skilful technical assistance. This work was supported by the grants of Ministry of Defense No. OBVLAJEP20032.

\section{References}

1. Cabal J, Bajgar J. Tabun - Reappearance 50 years later. Chem Listy 1999;93: 27-9. (in Czech)

2. Dohnal V, Kuča K, Havel J, Cabal J. Prediction of novel structures of efficient reactivators of cyclosarin-inhibited acetylcholinesterase using method of artificial neural networks. LIBLICE 2004 - Progresses in organic, bioorganic and pharmaceutic chemistry, Nymburk 28. - 30. 11. 2003, Poster presentation.

3. Kassa J, Cabal J. A comparison of the efficacy of acetylcholinesterase reactivators against cyclohexyl methylphosphonofluoridate (GF agent) by in vitro and in vivo methods. Pharmacol Toxicol 1999;84:41-5.

4. Kassa J, Cabal J. A comparison of the efficacy of a new asymmetric bispyridinium oxime $\mathrm{BI}-6$ with presently used oximes and $\mathrm{H}$ oximes against sarin by in vitro and in vivo methods. Hum Exp Toxicol 1999;18:560-5.

5. Kassa J, Cabal J. A comparison of the efficacy of a new asymmetric bispyridinium oxime BI- 6 with currently available oximes and $\mathrm{H}$ oximes against soman by in vitro and in vivo methods. Toxicology 1999;132:111-8.

6. Kassa J, Krejcova G. Neuroprotective Effects of Currently Used Antidotes in Tabun-Poisoned Rats. Pharmacology and Toxicology 2003;92:258-64

7. Kassa J, Krejčová G, Samnaljev I. A comparison of the neuroprotective efficacy of pharmacological pretreatment and antidotal treatment in soman-poisoned rats. Acta Med (Hradec Králové) 2003;46(3):101-7.

8. Kassa J, Kročová Z, Ševelová L, Sheshko V, Kasalová I, Neubauerová V. Low-level sarin-induced alteration of immune system reaction in inbred BALB/c mice. Toxicology 2003;187:195-203.

9. Krejcova G, Kassa J. Neuroprotective efficacy of pharmacological pretreatment and antidotal treatment in tabun-poisoned rats. Toxicology 2003;185: 128-39.

10. Kuča K, Bielavský J, Cabal J, Bielavska M. Synthesis of a potentesis of a potentof acetylcholinesterase - 1-(4-hydroxyiminomethylpyridinium)-3-(carbamoylpyridinium)-propane dibromide. Tetrahedron Lett 2003:44:3123-5.

11. Kuča K, Bielavský J, Cabal J, Kassa J. Synthesis of a new reactivator of tabun-inhibited acetylcholinesterase. Bioorg Med Chem. Lett 2003;13:3545-7.

12. Kuča K, Cabal J, Kassa J. A comparison of the efficacy of a bispyridinium oxime - 1,4-bis-(2-hydroxyiminomethylpyridinium) butane dibromide and currently used oximes to reactivate sarin, tabun or cyclosarin-inhibited acetylcholinesterase by in vitro methods. Pharmazie 2004 In press.

13. Kuča K, Cabal J, Patočka J, Kassa J. Synthesis of bisquaternary symmetric $-\chi, \delta$ bis(2-hydroxyiminomethylpyridinium)alkane dibromides and their reactivation of cyclosarin-inhibited acetylcholinesterase. Lett Org Chem 2004;1:84-6.

14. Kuča K, Dohnal V. 1,3-bis(2-hydroxyiminomethylpyridinium) propane as the potential reactivator of the acetylcholinesterase inhibited by nerve agents. Acta Med (Hradec Králové) 2004, In press.

15. Kuča K, Kassa J. A comparison of the ability of a new byispyridinium oxime 1-(4-hydroxyiminomethylpyridinium)-4-(4-carbamoylpyridinium)butane dibromide and currently used oximes to reactivate nerve agent-inhibited rat brain acetylcholinesterase by in vitro methods. J Enzyme Inhib Med Chem 2003;18: 529-35.

16. Kuča K, Kassa J. In Vitro reactivation of acetylcholineesterase using the oxime K027. Vet Hum Toxicol 2004,46:15-18.

17. Kuča K, Patočka J. Reactivation of cyclosarin-inhibited rat brain acetylcholinesterase by pyridinium-oximes. J. Enzime Inhib Med Chem 2004;19:39-43.

18. Kuča K, Patočka J, Cabal J. Reactivation of organophosphate inhibited acetylcholinesterase activity by $\alpha, \omega$-bis-(4-hydroxyiminomethylpyridinium)alkanes in vitro. J Appl Biomed 2003, 1:207-11.

19. Ševelová L., Vachek J. Effect of methoxime combined with anticholinergic, anticonvulsant or anti-HCN drugs in tabun-poisoned mice. Acta Medica (Hradec Králové) 2003, 46:109-12.

20. Zajtchuk R, Bellamy RF. Medical aspects of chemical and biological warfare. Office of the Surgeon General, Department of the Army, United states of America, 1997.

Submitted February 2004.

Accepted April 2004.

Ing. Kamil Kuča,

Purkyně Military Medical Academy,

Department of Toxicology,

Třebešská 1575, 50001 Hradec Králové,

Czech Republic.

e-mail: kucakam@pmfhk.cz 